hep-ph/0503052

March, 2005

\title{
Split Supersymmetry in Unified Models
}

\author{
Bhaskar Dutta and Yukihiro Mimura \\ Department of Physics, University of Regina, \\ Regina, Saskatchewan S4S 0A2, Canada
}

\begin{abstract}
In the context of split supersymmetry, the gaugino mass spectrum seems to be very important to satisfy the dark matter content of the universe and the gauge coupling unification. In this paper, we have considered various sources of gaugino masses in the context of unified models. We show that the gaugino mass spectrum varies in different unification pictures. In the context of SU(5), we have found that the Bino/Wino mass ratio can be close to one at the weak scale which is helpful to satisfy the WMAP data. The gluino/Wino mass ratio is also different from the usual scenario of unified gaugino masses. The gaugino masses can be around one $\mathrm{TeV}$ and $m_{\mathrm{SUSY}}$ is chosen so that the gluino mass does not create any cosmological problem. In the context of the Pati-Salam model, we show that the gluino mass can be made very heavy even after maintaining the unification of the gauge couplings.
\end{abstract}




\section{Introduction}

The determination of cosmological parameters [1] have impacts on particle physics. The revelation of the non-zero cosmological constant (responsible for dark energy) seems to be the biggest problem in particle physics [2]. It is expected that there should be a mechanism to tune the cosmological constant [3]. Such a mechanism may also be applied to solve the gauge hierarchy problem [4]. In this scenario the low energy supersymmetry (SUSY) is not a necessity for the physics beyond the standard model(SM). In another view point, the anthropic landscape of string/M-theory vacua has been discussed recently [5], and the naturalness principle does not have the criterion to select models in such an analysis. In fact, such a statistical argument of SUSY breaking vacua claims that the high energy SUSY breaking is preferred [6] when the cosmological constant is anthropically tuned to be zero and there exist multiple sources of SUSY breaking, $m_{3 / 2}^{2}=\left(\sum_{i}\left|F_{i}\right|^{2}+\sum_{i} D_{i}^{2}\right) / M_{P}^{2}$. The low energy theories have been discussed in the context of such anthropic landscape [7].

Under such circumstances, the split SUSY scenario [8, 9, 10, 11, 12, 13, 14] is suggested as one possibility of a phenomenological model. In the scenario, the SUSY breaking scale, $m_{\mathrm{SUSY}}$, is large, e.g. $10^{9} \mathrm{GeV}$, and the squarks and sleptons are heavy, while gauginos and Higgsinos remain light (masses $\sim$ weak scale) due to $R$-symmetry or other related symmetries. Of course, SUSY does not play an essential role to explain the gauge hierarchy, but other motivations of supersymmetry are maintained: e.g. the three gauge couplings of the SM are unified at the GUT scale, and the lightest neutralino is a candidate to explain the cold dark matter content

of the Universe. Since all the sfermions are assumed to be heavy, the FCNC related problems are absent and the lightest CP-even neutral Higgs mass can be large enough compared to the current experimental bound [8, 9]. The dangerous proton decay operators are also suppressed. Therefore, the typical fine-tuning problems of the low energy SUSY models are absent, except for the justification of the fact that one of the Higgs doublets is light [15].

In the phenomenological framework of the scenario, it is important to choose the SUSY breaking scale. The scale is written as $m_{\mathrm{SUSY}}=F_{X} / M_{P}$, where $F_{X}$ is an $F$-term of the chiral superfield $X$, which is a dominant source of SUSY breaking, and $M_{P}$ is the Planck scale. Since the gaugino masses can be assumed to be $m_{\mathrm{SUSY}}^{2} / M_{P}$ naively (if the dominant contribution is forbidden by $R$-symmetry), the typical scale of $m_{\mathrm{SUSY}}$ is $10^{10} \mathrm{GeV}$. However, the $m_{\mathrm{SUSY}}$ can be $10^{6} \mathrm{GeV}$ (PeV scale) [16] (in this case the anomaly mediation seems to be the dominant source for the gaugino masses), or can be much larger $\sim 10^{13} \mathrm{GeV}$ (in this case the gauginos are heavy and only Higgsinos masses are light to be at around the TeV scale). One can also consider the models with the Fayet-Iliopoulos D-terms [17] which generates another source of SUSY breaking. 

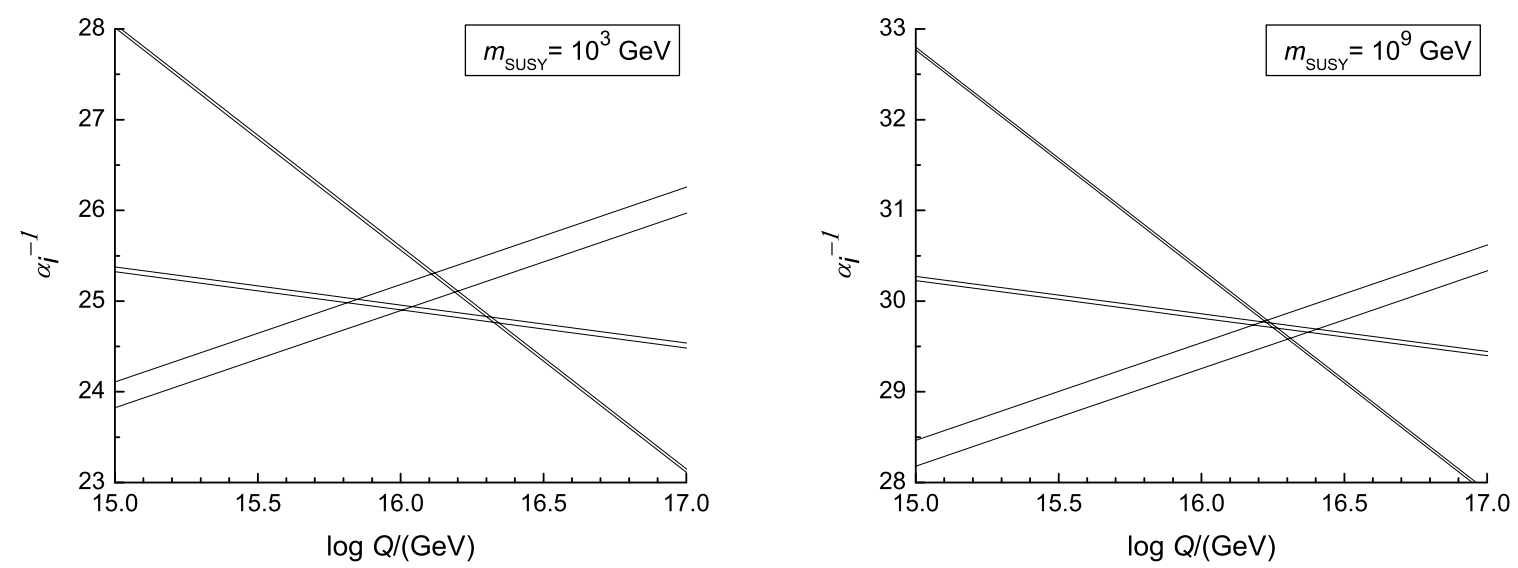

Figure 1: Two-loop RGE evolution around the GUT scale in the case $\tan \beta=10$. The lines are drawn at $1 \sigma$ range in the case $M_{3}=\mu=1 \mathrm{TeV}$ and $M_{2}=300 \mathrm{GeV}$.

Now we will clarify the motivation of our work in the context of split SUSY. First, let us investigate the gauge coupling unification. As it is well known, the three gauge couplings are unified at GUT scale with the following condition at one-loop level,

$$
\frac{12}{\alpha_{2}\left(M_{Z}\right)^{S M}}-\frac{5}{\alpha_{1}\left(M_{Z}\right)^{S M}}-\frac{7}{\alpha_{3}\left(M_{Z}\right)^{S M}}=-\frac{19}{4 \pi} \ln \frac{M_{S U S Y}}{M_{Z}}
$$

when all the SUSY particles (and a heavier Higgs doublet) are at a single scale $M_{S U S Y}$ and the possible GUT threshold corrections are neglected. The $M_{S U S Y}$ scale is calculated at around 50 $\mathrm{GeV}$ by this one-loop equation, but the scale is corrected to be around one TeV when two-loop contributions are included. The SUSY threshold correction in the Eq.(1) can be rewritten as a multi-scale threshold,

$$
-\frac{19}{4 \pi} \ln \frac{M_{S U S Y}}{M_{Z}} \rightarrow \frac{1}{2 \pi}\left(14 \ln \frac{M_{3}}{M_{2}}-2 \ln \frac{M_{2}}{M_{Z}}-6 \ln \frac{\mu}{M_{Z}}+\frac{3}{2} \ln \frac{m_{\tilde{q}}}{m_{\tilde{\ell}}}-\frac{3}{2} \ln \frac{m_{H}}{M_{Z}}\right)
$$

where $M_{3}, M_{2}$ are gluino and Wino masses, $\mu$ is a Higgsino mass, $m_{\tilde{q}}, m_{\tilde{\ell}}$ are squark and slepton masses, and $m_{H}$ is the mass of the heavier Higgs doublet. It is easy to see that the SUSY threshold correction is insensitive to the scalar masses, $m_{\tilde{q}}, m_{\tilde{\ell}}, m_{H}$, which we call $m_{\mathrm{SUSY}}$ in split SUSY case. Thus, even if those scalar masses are splitted to be heavy, the coupling unification can still hold. It is important to notice that the gluino/Wino mass ratio is a significantly sensitive parameter in the Eq.(2). In the case of usual mSUGRA models, the gluino/Wino mass ratio is about 3 at a $\mathrm{TeV}$ scale, and thus the strong gauge coupling is predicted to be larger at the $M_{Z}$ scale. In order to predict the correct magnitude of the strong coupling one needs $\mu$ to be $\sim 10 \mathrm{TeV}$, but such a direction is disfavored satisfying the WMAP data. In 
split SUSY, $m_{H}$ can compensate the discrepancy by the choice $m_{H} \sim 10^{9} \mathrm{GeV}$, and thus the Higgsino and the Wino can be kept at the TeV scale and these can be the sources of dark matter. In Fig.1, we show the 2-loop RGE evolutions of gauge couplings [18] in the $\overline{D R}$ scheme in the case, $M_{3}=\mu=1 \mathrm{TeV}$ and $M_{2}=300 \mathrm{GeV}$. In the figure, we use $\alpha_{3}^{\overline{M S}}\left(M_{Z}\right)=0.1187 \pm 0.002$, $\sin ^{2} \theta_{W}^{\overline{M S}}\left(M_{Z}\right)=0.23120 \pm 0.00015$, and $1 / \alpha^{\overline{M S}}\left(M_{Z}\right)=127.918 \pm 0.018$ [19].

Next, let us see the neutralino dark matter condition to obtain the dark matter density observed as by the WMAP data [1], $0.094<\Omega_{D M} h^{2}<0.129$. The Refs. [10, 20] use the FORTRAN DarkSUSY package [21] and find solutions which satisfy WMAP data in the case of split SUSY. The solutions are on the 2D surface in the $\left(M_{1}, M_{2}, \mu\right)$ space, and can be written as the following three typical cases:

1. Bino-like dark matter : $M_{1} \sim M_{2} \lesssim \mu$, or $\quad M_{1} \sim \mu \lesssim M_{2}$.

2. Wino dark matter : $M_{2} \simeq 2-2.5 \mathrm{TeV}, M_{2} \sim \mu \lesssim M_{1}$.

3. Higgsino dark matter : $\mu \simeq 1 \mathrm{TeV}$.

For the Bino dark matter case, the universe is overclosed when $M_{1} \ll M_{2}$, $\mu$. So the Bino must be mixed with Wino and/or Higgsino. When Higgsino is heavier than Bino and Wino, the Bino and Wino masses are needed to be degenerate, $M_{1} \simeq M_{2}$, to make the mixing large. In the Bino-like dark matter case above, the LSP neutralino mass should be less than around $1 \mathrm{TeV}$.

The gaugino/Higgsino spectrum in split supersymmetry is important to satisfy the WMAP data. In this paper, we will try to produce the above gaugino spectrum in the context of the unified models. We will see that the cases (1) and (2) can be observed in the context of unified models at the GUT scale e.g. $S U(5), S U(4)_{c} \times S U(2)_{L} \times S U(2)_{R}$ etc.

It has been pointed out [8] that the gluino is the most important particle since its lifetime can be a direct probe of the scale $m_{\mathrm{SUSY}}$. The gluino, in this model, can decay only through the virtual scalar quark and it is long-lived [22] compared to the usual low energy SUSY framework. The gluino decay rate of $\tilde{g} \rightarrow q \bar{q} \chi$ is given as [23]

$$
\Gamma \sim \frac{\alpha_{s} \alpha}{48 \pi} \frac{m_{\tilde{g}}^{5}}{m_{\tilde{q}}^{4}}\left(a_{j i}^{2}+b_{j i}^{2}\right),
$$

where the masses of quark and neutralino for final states are neglected compared to the gluino mass and $a_{j i}, b_{j i}$ are the neutralino-squark-quark couplings which are defined in the Ref. [23]. Since the gluino lifetime is sensitive to the scalar quark mass, and so the scalar mass scale can be probed from the lifetime of gluino. The lifetime of gluino is $1-10^{4}$ second when the gluino mass is $1 \mathrm{TeV}$ and scalar mass scale is $10^{9-10} \mathrm{GeV}$. But this will disturb the nucleosynthesis in the early universe. Since one of the motivation of this framework is also the explanation of 
the origin of cold dark matter, it is important to probe the lifetime of gluino. The lifetime can determine the allowed ranges of gluino and scalar quark masses in this framework. Here, we naively consider two typical cases to make the gluino lifetime to be around $10^{-7}$ second, which is the freeze-out time of cold dark matter in the neutralino LSP scenario: (A) the gluino mass is $1 \mathrm{TeV}$ and the scalar mass scale is $10^{7} \mathrm{GeV},(\mathrm{B})$ the gluino mass is heavier to be $10^{4.5} \mathrm{GeV}$ and the scalar mass scale is $10^{9} \mathrm{GeV}$. The gluino mass scale is the key issue to compare the phenomenological models which can be distinguished in the future collider experiments such as LHC and ILC. We will consider different unified models and show that the above conditions for the gluino mass can be satisfied. We will also show that the gluino mass can be very heavy in certain unified models.

In this paper, we discuss several sources of gaugino masses and point out a dominant source in the case of unified models. We discuss several unification scenarios and show that the gaugino spectrum is a useful probe of these scenarios. The Higgsino (for both charged and neutral) mass is characterized only by the mass parameter $\mu$, which is just a model parameter. It is hard to specify the symmetry that suppresses the $\mu$-term and the reason for one of the Higgs doublets being light [15]. Thus observing a Higgsino is not a good probe to determine the physics at the high energy scale. For gauginos, on the other hand, we have three mass parameters, and a given gaugino spectrum can be related to a particular unified model. Therefore the observation of the gaugino spectrum can be a good probe of the theory at a high energy scale. In section 2, we discuss the origins of gaugino masses, and point out that the Goldstino contribution may important. We discuss the SU(5) unification scenario in section 3, and in section 4 we discuss $\mathrm{SU}(4)_{c} \times \mathrm{SU}(2)_{L} \times \mathrm{SU}(2)_{R}, \mathrm{SU}(3)_{c} \times \mathrm{SU}(2)_{L} \times \mathrm{SU}(2)_{R} \times \mathrm{U}(1)_{B-L}$ and $\mathrm{SO}(10)$ models and we conclude in section 5 .

\section{Possible Origins of Gaugino Masses}

As we have seen in the introduction, the gaugino and the Higgsino mass spectrums are important for gauge coupling unification, neutralino dark matter, and also for the measurements at the colliders. Since the magnitudes of three gaugino masses are related to the origin of the gaugino masses, it is worth classifying the origins. In this section, we enumerate the possible origins of gaugino masses. 


\subsection{The usual mSUGRA origin of gaugino mass}

The gaugino mass in the superfield formalism is written as

$$
\int d^{2} \theta\left(\frac{1}{g^{2}}+M_{1 / 2} \theta^{2}\right) W^{\alpha} W_{\alpha}+\text { H.c. },
$$

where $g$ is the gauge coupling constant. In the unified model such as SU(5), the gaugino masses are unified at the GUT scale just like the gauge coupling constants. We assume that the contribution from the SUSY breaking, $M_{1 / 2} \sim F_{X} / M_{P} \sim m_{\mathrm{SUSY}}$, is forbidden by the $R$ symmetry, and the leading contribution is $M_{1 / 2} \sim F_{X} F_{X}^{\dagger} / M_{P}^{3} \sim m_{\text {SUSY }}^{2} / M_{P}$. As usual, the three gaugino masses are unified at the GUT scale also in the split SUSY scenario. However, the usual relation in mSUGRA, $M_{i}=\alpha_{i} M_{1 / 2}$, is broken below $m_{\mathrm{SUSY}}$ even at the 1-loop level. The RGE evolution below $m_{\mathrm{SUSY}}$ is described in Ref.[10].

\subsection{The Anomaly mediation}

Even if the gauge field strength $W_{\alpha}$ does not couple to the spurion singlets, the anomaly mediation 24] can produce gaugino masses by a chiral compensator field $\phi$ in supergravity,

$$
M_{i}=\frac{\beta\left(g_{i}\right)}{g_{i}} F_{\phi}
$$

If this contribution is dominant as a source for gaugino masses, Wino can be the dark matter candidate and $M_{2} \simeq 2-2.5 \mathrm{TeV}$. Then the scalar mass scale is around $10^{6} \mathrm{GeV}[16$. The relation (5) is also modified below $m_{\mathrm{SUSY}}$ [10]. Since the gluino/Wino mass ratio is large $\left(M_{3} / M_{2} \sim 8-9\right)$, the Higgsino needs to be heavy $\mu \sim 20 \mathrm{TeV}$ to satisfy the gauge coupling unification. Thus, every superparticle is heavy in this solution.

\subsection{Gauge mediation}

When the messenger fields $f, \bar{f}$ couple to the spurion singlet,

$$
\int d^{2} \theta\left(m+\theta^{2} F_{m}\right) f \bar{f}+H . c .,
$$

the gauginos masses are produced at the one-loop finite contribution [25],

$$
M_{i}=\frac{\alpha_{i}}{4 \pi} T_{i}(f) m\left(\frac{M_{f 1}^{2}}{M_{f 1}^{2}-m^{2}} \ln \frac{M_{f 1}^{2}}{m^{2}}-\frac{M_{f 2}^{2}}{M_{f 2}^{2}-m^{2}} \ln \frac{M_{f 2}^{2}}{m^{2}}\right) \sin 2 \beta_{m},
$$

where $T_{i}(f)$ is Dynkin index for $f+\bar{f}$ and $M_{f i}^{2}$ are the scalar messenger eigenmasses

$$
M_{f 1,2}^{2}=m^{2}+\frac{m_{f}^{2}+m_{\bar{f}}^{2}}{2} \pm \sqrt{\left|F_{m}\right|^{2}+\left(\frac{m_{f}^{2}-m_{\bar{f}}^{2}}{2}\right)^{2}}
$$


and $\tan 2 \beta_{m}=2\left|F_{m} /\left(m_{f}^{2}-m_{\bar{f}}^{2}\right)\right|$. The $m_{f}^{2}, m_{\bar{f}}^{2}$ are the SUSY breaking masses for messenger scalars.

When the messenger scale is much larger than the SUSY breaking scale, $m^{2} \gg F_{m}, m_{\mathrm{SUSY}}^{2}$, the usual gauge mediation formula at the messenger scale $m$ is

$$
M_{i}=\frac{\alpha_{i}}{4 \pi} T_{i}(f) \frac{F_{m}}{m} .
$$

If the messenger scale is much less than $m_{\mathrm{SUSY}}$, the gaugino masses are proportional to $m F_{m} / m_{\mathrm{SUSY}}^{2}$ instead of $F_{m} / m$.

\section{$2.4 \mu$-term}

Since the $B$-term is large in the case of split SUSY, the Higgs doublet can work as messenger field. But, since one of the Higgs scalar is splitted to be light by the fine-tuning, $B \mu \sim$ $\mu^{2}+m_{\text {SUSY }}^{2}$, the usual formula of gauge mediation, Eq.(9), can not be applied.

The wino and the bino masses can be generated from $\mu$ by finite Higgsino-Higgs one-loop corrections. In the case $m_{\mathrm{SUSY}} \gg \mu \gg M_{W}$, we have

$$
M_{2} \simeq \frac{\alpha_{2}}{4 \pi} \mu \sin 2 \beta \ln \frac{m_{\mathrm{SUSY}}^{2}}{\mu^{2}}, \quad M_{1} \simeq \frac{\alpha^{\prime}}{4 \pi} \mu \sin 2 \beta \ln \frac{m_{\mathrm{SUSY}}^{2}}{\mu^{2}} .
$$

We also have one-loop RGE corrections below $m_{\text {SUSY }}$ due to the loop diagram where the light Higgs doublet propagates in the loop. The RGE correction gives rise to same signature in the finite correction in Eq.(10). Finally, we have a gaugino mass from $\mu$-term contribution below $\mu$,

$$
M_{2} \simeq \frac{\alpha_{2}}{2 \pi} \mu \sin 2 \beta \ln \frac{m_{\mathrm{SUSY}}^{2}}{\mu^{2}}, \quad M_{1} \simeq \frac{\alpha^{\prime}}{2 \pi} \mu \sin 2 \beta \ln \frac{m_{\mathrm{SUSY}}^{2}}{\mu^{2}} .
$$

However, this contribution (just by itself) is not favored if neutralino is the dark matter candidate, since the bino nature of the LSP may overclose the universe. We need another source of gaugino masses to make $M_{2} \sim M_{1}$ or $M_{2} \ll M_{1}$ in the case $M_{1,2} \ll \mu$. The possibility of heavy Higgsino is studied in Ref.[26].

\subsection{The $A$-term}

\subsubsection{The scalar trilinear couplings for Sfermions}

The Sfermion trilinear couplings $\left(A_{f} \tilde{q} \tilde{u}^{c} h_{u}\right)$ generates gaugino masses through two-loop RGE 27] above $m_{\mathrm{SUSY}}$.

Since the $A_{f}$ term breaks $R$-symmetry, one can forbid this contribution in the split SUSY scenario in the same way as original gaugino masses are forbidden. However, if one protects the 
gaugino (and/or Higgsino) masses by an anomalous $U(1)$ symmetry, but breaks $R$-symmetry, this contribution can be dominant [28].

When only the top Yukawa contribution is dominant, the gaugino masses at the SUSY breaking scale are approximately:

$$
M_{i} \sim 2 c_{i} \frac{\alpha_{i} \alpha_{t}}{(4 \pi)^{2}} A_{t} \ln \frac{M_{G}}{m_{\mathrm{SUSY}}},
$$

where $\alpha_{t}=y_{t}^{2} /(4 \pi), c_{i}=(26 / 5,6,4)$ and $A_{t}$ is the $A$-parameter for top-stop-Higgs coupling. In this case, the Bino is the lightest among the gauginos at the weak scale. So when this contribution is dominant, the Higgsino mass needs to be chosen appropriately to satisfy the WMAP data.

\subsubsection{The Goldstino contribution}

In the super Higgs mechanism, the Goldstino $\widetilde{\Sigma}$ is the Higgsino eaten by the heavy gaugino. The Goldstinos acquire masses at the symmetry breaking scale by folding the Dirac mass with heavy gauginos, $\lambda_{X}$, corresponding to the broken generators of gauge symmetry. In the SUSY limit,

the bilinear terms of Goldstinos are absent since their superpartners (the would-be Goldstone bosons) are massless. If the SUSY is broken in the GUT Higgs potential, the Goldstino bilinear term is non-zero,

$$
\delta m \sim A_{\mathrm{GUT}}+\frac{m_{\mathrm{SUSY}}^{2}}{M_{\mathrm{GUT}}},
$$

where $A_{\mathrm{GUT}}$ is a dimension one SUSY breaking parameter in the GUT Higgs potential. Then the heavy gauginos become pseudo-Dirac fields with following the mass terms

$$
\left(\lambda_{X}, \widetilde{\Sigma}_{X}\right)\left(\begin{array}{cc}
M_{1 / 2} & M_{V} \\
-M_{V} & \delta m
\end{array}\right)\left(\begin{array}{c}
\lambda_{\bar{X}} \\
\widetilde{\Sigma}_{\bar{X}}
\end{array}\right)+H . c .,
$$

and the heavy gaugino loop diagram, as shown in Fig 2, generates the finite corrections to the masses for the SM gauginos [29],

$$
M_{i}=\frac{\alpha_{G}}{2 \pi} T_{i}\left(\Sigma_{X}\right) \delta m
$$

We also have the contribution which is proportional to $M_{1 / 2}$ as a one-loop correction to the original gaugino mass $M_{1 / 2}$.

The MSSM superpotential can be invariant under the $R$-symmetry and thus the $A_{f}$ contribution can be always controlled by $R$-symmetry. The other contributions listed above also can be forbidden by $R$-symmetry. On the other hand, the realistic GUT Higgs superpotential itself breaks $R$-symmetry, and thus the GUT symmetry breaking always causes the $R$-symmetry 


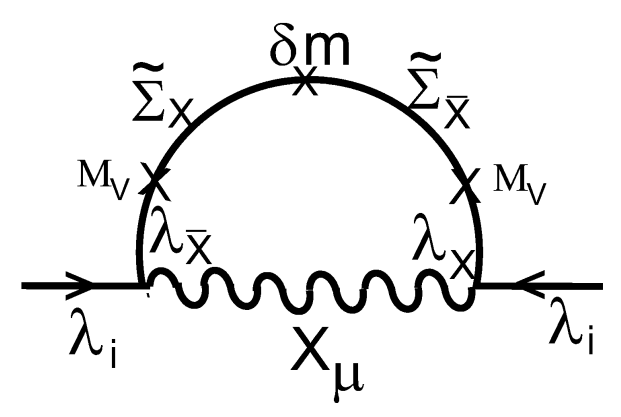

Figure 2: One-loop diagram which gives the finite correction to the gaugino masses.

breaking and contributes to the gaugino masses through the Goldstino loop. Consequently, this may introduce a dominant contribution to the gaugino mass in the split SUSY scenario as long as the unified symmetry is broken by Higgs mechanism, unless we introduce some other symmetries to control it.

Note that the $m_{\mathrm{SUSY}}$ in Eq.(13) is the scalar mass for GUT Higgs fields. In order to keep the gaugino masses less than $1 \mathrm{TeV}$, we need $m_{\mathrm{SUSY}} \lesssim 10^{11} \mathrm{GeV}$ when $M_{\mathrm{GUT}} \sim 10^{16} \mathrm{GeV}$.

\subsection{Selection of the origins}

All these above contributions can be present in the gaugino masses and any one of the above sources can generate dominant contribution. The parameter space of $\left(M_{1}, M_{2}, \mu\right)$ is restricted by phenomenologies. For example, if the Higgsinos are much heavier than the gauginos, e.g. $\mu \simeq 10 \mathrm{TeV}$, we need $M_{2} \lesssim M_{1}$ and the wino-like neutralino is the LSP so that we do not exceed the amount of relic density [20]. Therefore, in the heavy Higgsino case, the usual mSUGRA source is not favored very much and the anomaly mediation is a good candidate. In the case of anomaly mediation, $M_{2} \sim 2-2.5 \mathrm{TeV}$ is required for the Wino dark matter. The gravitino and the scalar masses are about $10^{6} \mathrm{TeV}$ [16]. If the Higgsinos are light, the Higgsinolike neutralino can be the LSP. However, in this case, the masses of lightest neutralino and chargino are degenerate if both $M_{1}$ and $M_{2}$ are large and the relic density can be very small due to the coannihilation processes. In order to satisfy the WMAP data, the Higgsino should be around $1 \mathrm{TeV}[20$.

If the origin of gaugino masses produces the three gaugino mass parameters of the same order, the gluino mass is less than several $\mathrm{TeV}$ (since the dark matter constraint require the other gaugino masses to be $\sim 1 \mathrm{TeV}$ ). This means that the lower scale of SUSY breaking is favored due to the lifetime of the gluino as we have discussed in the introduction. So, the anomaly mediation with the PeV scale $m_{\mathrm{SUSY}}$ may be a good scenario. However, all the superparticles need to be heavier than $2 \mathrm{TeV}$ and such a situation is not very attractive for the 
precision electroweak data. On the other hand, if the origin of the gauginos masses is due to the Goldstino contribution, which happens in the case of the unifying models, the gaugino spectrum depends on the unification group. The precise dependence of the spectrum on different unifying gauge symmetries are discussed in the next two sections.

\section{$3 \mathrm{SU}(5)$}

The $A$-term in the GUT Higgs potential as shown in the Eq.(13) can be a dominant contribution of gaugino masses in split SUSY scenario. In this section, we study the contribution in the SU(5) GUT model. The SU(5) symmetry is broken down to SM gauge group by the VEV of adjoint Higgs field $\Sigma$. The adjoint Higgs field is decomposed as

$$
\Sigma: \mathbf{2 4}=\Sigma_{8}:(\mathbf{8}, \mathbf{1})_{0}+\Sigma_{3}:(\mathbf{1}, \mathbf{3})_{0}+\Sigma_{1}:(\mathbf{1}, \mathbf{1})_{0}+\Sigma_{X}:(\mathbf{3}, \mathbf{2})_{-5 / 6}+\Sigma_{\bar{X}}:(\overline{\mathbf{3}}, \mathbf{2})_{5 / 6}
$$

When the singlet $\Sigma_{1}$ get $\operatorname{VEV}\left(\left\langle\Sigma_{1}\right\rangle=\sigma\right)$, the would-be Goldstone bosons, $\Sigma_{X, \bar{X}}$, are eaten by heavy gauge bosons, and their superpartners, Goldstinos $\tilde{\Sigma}_{X, \bar{X}}$, combine Dirac masses with heavy gauginos, $\lambda_{X, \bar{X}}$.

The Goldstino bilinear mass term is $(M-\lambda \sigma / \sqrt{30}) \tilde{\Sigma}_{X} \tilde{\Sigma}_{\bar{X}}$ for the Higgs superpotential which breaks $\mathrm{SU}(5)$ symmetry

$$
W=\frac{M}{2} \operatorname{Tr} \Sigma^{2}+\frac{\lambda}{3} \operatorname{Tr} \Sigma^{3}
$$

In the SUSY limit, the VEV is $\sigma=\sigma_{0}=\sqrt{30} M / \lambda$, and the bilinear term of the Goldstino is of course absent. However, if we include the soft SUSY breaking terms

$$
V_{\text {soft }}=m_{\Sigma}^{2} \operatorname{Tr} \Sigma \Sigma^{\dagger}+\left(\frac{1}{2} B_{G} M \operatorname{Tr} \Sigma^{2}+\frac{1}{3} \lambda A_{G} \operatorname{Tr} \Sigma^{3}+\text { H.c. }\right),
$$

the VEV shifts to $\sigma_{0}+\Delta \sigma$. Then the Goldstino bilinear mass $\delta m$ arises

$$
\delta m=-\frac{\lambda}{\sqrt{30}} \Delta \sigma \simeq B_{G}-A_{G}+\frac{m_{\Sigma}^{2}}{M},
$$

and the one-loop diagram in which heavy gauge bosons and gauginos propagate in Fig.2 induces the gaugino masses $M_{i}$ at GUT scale,

$$
M_{i}=\frac{\alpha_{G}}{2 \pi} T_{i}\left(\Sigma_{X}\right) \delta m
$$

Since the heavy multiplet, $(\mathbf{3}, \mathbf{2})_{-5 / 6}+(\overline{\mathbf{3}}, \mathbf{2})_{5 / 6}$, are non-singlets under each gauge symmetry, it contributes to all gaugino masses $M_{i}$, and the ratios at the GUT scale are

$$
M_{1}: M_{2}: M_{3}=5: 3: 2 .
$$


Since this ratio is different from the original unified gaugino source or anomaly mediation, one can distinguish this scenario from the measurement of the gaugino mass spectrum.

We note that the Goldstino contribution is not related to the details of the GUT superpotential. The gaugino mass ratios depends only on the quantum numbers of the broken generators. So even if we employ 75 Higgs to break SU(5) down to the SM, the mass ratio (21) does not change as long as the unified group is $\mathrm{SU}(5)$.

Let us see how the Goldstino contribution can dominate over the others. We assume that the spurion fields $X$ is the dominant source of the SUSY breaking. We assign a $R$-charge -1 to other spurion $S$ field and 1 to $\Sigma$, so that the superpotential $W=M \Sigma^{2} / 2+S \Sigma^{3} / M_{P}$ can be $R$-symmetric. We assign a positive and fractional $R$-charge to the spurion $X$ in order not to disturb the superpotential. Then the coupling $\lambda$ in Eq.(17) is $\lambda=3 S / M_{P}$ and the $A$ parameter is $A_{G}=F_{S} / S$, and $A_{G}$ is a free parameter $\left(A_{G}<m_{\text {SUSY }}\right.$ by assumption). We can suppress all other sources of gaugino masses as is done in the case of split SUSY. For example, if the gluino mass is around a $\mathrm{TeV}$ from the Goldstino contribution, $m_{\mathrm{SUSY}}$ is around $10^{7} \mathrm{GeV}$ for the cosmological reason. The usual mSUGRA leading contribution $M_{i} \sim m_{\mathrm{SUSY}}$ is forbidden by the $R$-symmetry, and thus the direct SUGRA contribution is $m_{\mathrm{SUSY}}^{2} / M_{P} \sim 10^{-4} \mathrm{GeV}$. Since the $R$-breaking is incorporated in the GUT symmetry breaking, the dimension one contribution from the SUSY breaking spurion $S$ with the one-loop factor can dominate the gaugino masses.

In this example, we have used $R$-symmetry to control the superpotential. Note that if the $R$-symmetry is the exact symmetry of the Lagrangian, the $R$-axion may cause the cosmological problem. Since we are not specifying the hidden sector potential, this concern is beyond the scope of the present paper. However, if the axion-like problem is considered seriously, one can construct models by using anomalous $\mathrm{U}(1)$ gauge symmetry in a similar way.

Note that if $B_{G}$ is large, the heavy chiral multiplets such as $\Sigma_{8}$ and $\Sigma_{3}$ work as messengers and they contribute to gaugino masses. In the above example of $R$-charge assignment, the magnitude of the $B$-term is $B_{G} M \sim m_{\text {SUSY }}^{2}\left(S / M_{P}\right)^{2}$, and thus $B_{G}$ itself is at most around weak scale in the split SUSY context and the messenger contribution is not very large. If we also make the adjoint Higgs mass $M$ to be the spurion mass and reassign $R$-charges appropriately, $B_{G}$ becomes free parameter $\sim F_{M} / M$ and such messenger effects can contribute to the gaugino masses. This $B$-term contribution depends on the details of the GUT particle spectrum and if this contribution dominates the gaugino mass spectrum will not exhibit the pattern of Eq.(21).

We now show the gaugino mass ratios when the gluino mass is $1 \mathrm{TeV}$ in Fig 3, The ratio depends on the SUSY breaking scale since the relation $\left(M_{i} / \alpha_{i}=\right.$ const.) is broken even at a one-loop RGE below $m_{\mathrm{SUSY}}$. The dark blue lines are for the usual ratio $M_{1}: M_{2}: M_{3}=1: 1: 1$ at GUT scale and red lines are for the Goldstino contribution $M_{1}: M_{2}: M_{3}=5: 3: 2$. The 


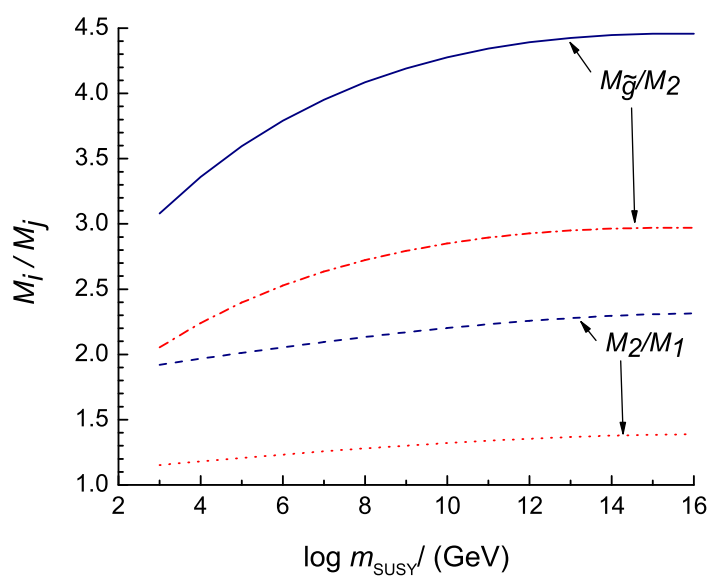

Figure 3: Gaugino mass ratios for the unified gaugino case (dark blue lines) and for the case that Goldstino contribution dominates (red lines). The values are given in the case where gluino mass is $1 \mathrm{TeV}$.

gluino mass is shown as a pole mass in $\overline{D R}$ scheme. It is interesting that the Wino/Bino mass ratio $M_{2} / M_{1}$ is close to one in the case of dominating Goldstino contribution. This is favored as a solution of Bino-like dark matter to satisfy the WMAP data. In this Bino-like dark matter with $M_{1} \sim M_{2}$, the condition is $100 \mathrm{GeV} \lesssim M_{1} \lesssim 1 \mathrm{TeV}$ and $\mu \gtrsim M_{1}$. In the case of the Wino and the Higgsino type dark matter, the Wino and the Higgsino masses are larger than $1 \mathrm{TeV}$. Thus the Bino-like dark matter prediction due to the SU(5) Goldstino contributions is attractive, since the dark matter candidate and the NLSP can be light and therefore, can be measured at the LHC.

If the Bino/Wino mass ratio is around one, the mass difference $(\Delta M)$ between the $\tilde{\chi}_{1}^{ \pm}$and the $\tilde{\chi}_{1}^{0}$ is very small (the precise value depends on $\mu$ ). Depending on the mass difference, the final states of a $\tilde{\chi}_{1}^{ \pm}$decay will contain lepton plus missing energy (arising from $\nu$ and $\tilde{\chi}_{1}^{0}$ ) and $q q^{\prime}$ plus missing energy (for very small $\Delta M$, there will be pions) or stable tracks [30]. The final state particles are soft. An accurate mass measurement becomes difficult. In the case of $\tilde{\chi}_{2}^{0}$, the dependence of $\mu$ is also quite significant. The mass difference between the $\tilde{\chi}_{2}^{0}$ and the $\tilde{\chi}_{1}^{0}$ depends on $\mu$ crucially. We will have $l \bar{l}$ plus missing energy and $q \bar{q}$ plus missing energy in the final states and depending on the mass difference between $\tilde{\chi}_{1}^{0}$ and $\tilde{\chi}_{2}^{0}$, the final state particles could be very low in energy. As before, an accurate mass measurement becomes difficult. It is also possible that the $\tilde{\chi}_{2}^{0}$ may not decay inside the detectors (if $\mu$ is quite large). It is interesting to investigate all these final states at LHC [31]. 


\section{Other Unification Groups}

The gaugino mass spectrum from the Goldstino contributions does not depend on the details of the Higgs potential, but it rather depends on the quantum number of the broken generators for the Goldstino contribution. Therefore, if this Goldstino contribution dominates, the gaugino mass spectrum can be a good probe of how the SM gauge group is unified.

In the $\mathrm{SU}(5)$ Goldstino contribution, the broken generators corresponds to $(\mathbf{3}, \mathbf{2})_{-5 / 6}+$ $(\overline{3}, 2)_{5 / 6}$ and thus all three gauginos acquire masses at the same order. So, as long as the neutralino relic density dominates dark matter, the gluino mass should be around one $\mathrm{TeV}$, and the SUSY breaking scale will be at the PeV scale to make the gluino lifetime shorter.

In this section, we will study the partial unification group of the SM and other possible unification scenarios. The partial unification groups are $G_{3221}=\mathrm{SU}(3)_{c} \times \mathrm{SU}(2)_{L} \times \mathrm{SU}(2)_{R} \times$ $\mathrm{U}(1)_{B-L}$, and $G_{422}=\mathrm{SU}(4)_{c} \times \mathrm{SU}(2)_{L} \times \mathrm{SU}(2)_{R}$.

In the case of $G_{3221}$, the Goldstino which is non-singlet under the SM is $(\mathbf{1}, \mathbf{1})_{ \pm 1}$. Thus only the Bino mass $M_{1}$ is influenced by the Goldstino contribution and hence it is heavy. In this case, the neutralino dark matter can be either the Wino or the Higgsino type. Since the $G_{3221}$ breaking scale $M_{3221}$ can be much smaller than GUT scale, the Goldstino bilinear mass can be large even if $A, B$-terms are small, $\delta m \sim m_{\mathrm{SUSY}}^{2} / M_{2231}$.

In the case of $G_{422}$, the non-singlet Goldstinos are $(\mathbf{3}, \mathbf{1})_{2 / 3}+(\overline{\mathbf{3}}, \mathbf{1})_{-2 / 3}$ in addition to $(\mathbf{1}, \mathbf{1})_{ \pm 1}$. Therefore, the $G_{422}$ symmetry breaking contributes to both gluino and Bino masses and hence they are heavy. But the Wino mass does not have this contribution and hence can be light. This situation is suitable for the Wino LSP. Since the gluino can be heavy, the $m_{\mathrm{SUSY}}$ can be large $\sim 10^{9} \mathrm{GeV}$ even if the lifetime of the gluino is constrained. For example, we can consider a hierarchical spectrum, $M_{3} \sim 10^{8} \mathrm{GeV}, M_{1} \sim 10^{7} \mathrm{GeV}, \mu \sim 10^{4} \mathrm{GeV}$, and $M_{2}$ can be $\sim \mathrm{TeV}$ scale. We note that $\mu$ is generated from $M_{1}$ through the one-loop RGE below $m_{\mathrm{SuSY}}$, and $M_{2}$ can be generated from $M_{3}$ at the 3-loop order by the RGE effect above $m_{\mathrm{SUSY}}$.

The gauge coupling unification is not good when $M_{3} \gg M_{2}$. However we can use the light colored Higgs fields $\left((\mathbf{3}, \mathbf{1})_{1 / 3}+(\overline{\mathbf{3}}, \mathbf{1})_{-1 / 3}\right)$ to help unify the gauge couplings. Since $m_{\text {SuSY }}$ can be $\sim 10^{9} \mathrm{GeV}$, the proton decay operator mediated by the colored Higgsino exchange is not dangerous. The colored Higgsino masses can be generated by $M_{3}$ at 1-loop $\sim 10^{7} \mathrm{GeV}$ and the three gauge couplings are unified as shown in Fig团. The gauge couplings unify at $(3-6) \times 10^{15}$ $\mathrm{GeV}$.

In the case of flipped SU(5), the gaugino mass ratios at the GUT scale are

$$
M_{1}: M_{2}: M_{3}=1 / 5: 3: 2 \text {. }
$$

So the ratio of the Bino mass to the Wino mass at the weak scale is 1:30. The Bino mass is 


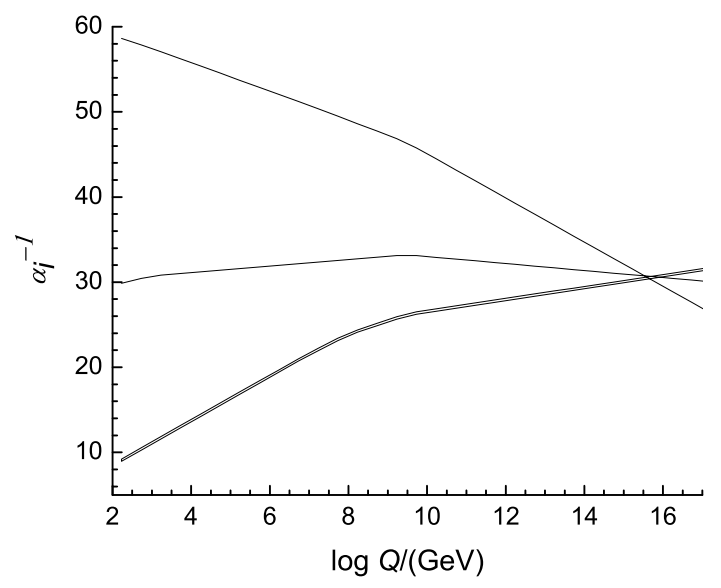

Figure 4: Gauge coupling running (two-loop) in the case where the $G_{422}$ Goldstino contributions are large. The gluino mass is assumed to be $10^{8} \mathrm{GeV}$. The colored Higgs fields are added to generate the unification.

the lightest and this situation is not good for dark matter unless the value of $\mu$ is around the bino mass. The gluino/Wino mass ratio however is same as in the $\mathrm{SU}(5)$ case.

The partial unification groups can be unified to $\mathrm{SO}(10)$. The broken generators in the $\mathrm{SO}(10)$ are $(\mathbf{3}, \mathbf{2})_{-5 / 6}+(\mathbf{3}, \mathbf{2})_{1 / 6}+(\overline{\mathbf{3}}, \mathbf{1})_{-2 / 3}+(\mathbf{1}, \mathbf{1})_{1}+$ c.c. using the SM quantum numbers. The first one corresponds to the $\mathrm{SU}(5)$ broken generator and the second one corresponds to the flipped-SU(5) broken generator. The other terms correspond to the $G_{422}$. Each broken generator can contribute to the gaugino mass spectrum. Depending on the Goldstino spectrum, each contribution can contribute coherently or some of the contributions are dominant. For example, in the limit that each multiplet contributes equally, we find the ratio at the GUT scale: $M_{1}: M_{2}: M_{3}=8: 6: 5$, and again it is different from the unified scenario. It is interesting that the measurement of the gaugino mass spectrum at the colliders will tell us about the broken generators.

\section{Conclusion}

The gaugino and the Higgsino masses are important for the motivations of SUSY which accommodates the gauge coupling unification and the neutralino dark matter. In the split SUSY scenario, it is assumed that only the gauginos and the Higgsinos are splitted to be light and their masses at the low energy scale are important to realize the motivation of SUSY.

In this paper, we have considered various sources of gaugino masses. Among them, we focus on the Goldstino contributions which arise in the case of unified gauge symmetry models. In the 
usual supergravity models, the unified gaugino mass at GUT scale is the leading contribution, and the Goldstino contribution is a higher order correction. However, if the original unified gaugino masses suppressed to be $F_{X} F_{X}^{\dagger} / M_{P}^{3}$ by $R$-symmetry (or other related symmetries), the Goldstino contribution may dominate. This is because the GUT superpotential usually breaks the $R$-symmetry. The GUT symmetry breaking requires the $R$-symmetry breaking, and generates a gaugino mass $\sim F_{X} / X$ with one-loop factor. The Goldstino contribution can dominate, as long as we consider the unified models in the split SUSY scenario with gauge symmetry being broken by the Higgs mechanism.

Interestingly, the Goldstino contribution depends only on the quantum numbers of the broken generators. Thus the gaugino mass spectrum is predictive especially in the $\mathrm{SU}(5)$ case. As we have shown in Fig 3 , the Wino and Bino masses are almost same $M_{1} \simeq M_{2}$ contrary to the mSUGRA case, $M_{2} / M_{1} \simeq 2$ at the weak scale. This is favored for the Bino-like dark matter in the split SUSY model to satisfy the recent WMAP data. The other dark matter solutions, the Higgsino or the Wino dark matter scenarios require the LSP mass $\gtrsim 1 \mathrm{TeV}$, whereas the Bino-like LSP mass can be less than a $1 \mathrm{TeV}$. Thus this prediction is attractive for the precision electroweak data and for the future measurements at LHC. Further, in this scenario, the gluino does not create any problem in the cosmological context since the SUSY breaking scale can be $\lesssim 10^{7} \mathrm{GeV}$. In the scenarios where the Bino and the Wino mass are very close or Wino is the LSP, we have soft jets, leptons in the final states of $\tilde{\chi}^{ \pm}, \tilde{\chi}_{2}^{0}$ decays.

We also considered other partial unification groups. In the case of $G_{422}$, only the Wino mass can be light among the three gauginos. In this case, the gluino can be heavy and harmless even if the SUSY breaking scale is large. In this scenario, the WMAP data is satisfied by the Wino dark matter.

\section{Acknowledgments}

This work is supported by the Natural Sciences and Engineering Research Council of Canada.

\section{References}

[1] S. Perlmutter et al. [Supernova Cosmology Project Collaboration], Astrophys. J. 517, 565 (1999) astro-ph/9812133; D. N. Spergel et al. [WMAP Collaboration], Astrophys. J. Suppl. 148, 175 (2003) astro-ph/0302209.

[2] S. Weinberg, Rev. Mod. Phys. 61, 1 (1989). 
[3] R. Bousso and J. Polchinski, JHEP 0006, 006 (2000) hep-th/0004134; J. L. Feng, J. March-Russell, S. Sethi and F. Wilczek, Nucl. Phys. B 602, 307 (2001) hep-th/0005276; S. Kachru, R. Kallosh, A. Linde and S. P. Trivedi, Phys. Rev. D 68, 046005 (2003) hep-th/0301240.

[4] E. Silverstein, hep-th/0407202.

[5] L. Susskind, hep-th/0302219; M. R. Douglas, JHEP 0305, 046 (2003) hep-th/0303194; hep-ph/0401004 F. Denef and M. R. Douglas, JHEP 0405, 072 (2004) hep-th/0404116; hep-th/0411183.

[6] L. Susskind, hep-th/0405189; M. R. Douglas, hep-th/0405279; Comptes Rendus Physique 5, 965 (2004) hep-th/0409207. M. Dine, E. Gorbatov and S. Thomas, hep-th/0407043

[7] N. Arkani-Hamed, S. Dimopoulos and S. Kachru, hep-th/0501082 M. Dine, D. O'Neil and Z. Sun, hep-th/0501214

[8] N. Arkani-Hamed and S. Dimopoulos, hep-th/0405159 N. Arkani-Hamed, S. Dimopoulos, G. F. Giudice and A. Romanino, Nucl. Phys. B 709, 3 (2005) hep-ph/0409232.

[9] A. Arvanitaki, C. Davis, P. W. Graham and J. G. Wacker, Phys. Rev. D 70, 117703 (2004) hep-ph/0406034; R. Mahbubani, hep-ph/0408096; M. Binger, hep-ph/0408240.

[10] G. F. Giudice and A. Romanino, Nucl. Phys. B 699, 65 (2004) [Erratum-ibid. B 706, 65 (2005)] hep-ph/0406088.

[11] B. Mukhopadhyaya and S. SenGupta, Phys. Rev. D 71, 035004 (2005) hep-th/0407225; N. Haba and N. Okada, hep-ph/0409113 hep-ph/0502213 G. M. Vereshkov, V. A. Beylin, V. I. Kuksa and R. S. Pasechnik, hep-ph/0410043 D. A. Demir, hep-ph/0410056; R. Allahverdi, A. Jokinen and A. Mazumdar, Phys. Rev. D 71, 043505 (2005) hep-ph/0410169; B. Bajc and G. Senjanovic, hep-ph/0411193; M. A. Diaz and P. F. Perez, hep-ph/0412066; L. Senatore, hep-ph/0412103 A. Datta and X. Zhang, hep-ph/0412255 S. P. Martin, K. Tobe and J. D. Wells, hep-ph/0412424 S. Kasuya and F. Takahashi, hep-ph/0501240, K. Huitu, J. Laamanen, P. Roy and S. Roy, hep-ph/0502052.

[12] S. h. Zhu, Phys. Lett. B 604, 207 (2004) hep-ph/0407072; W. Kilian, T. Plehn, P. Richardson and E. Schmidt, Eur. Phys. J. C 39, 229 (2005) hep-ph/0408088; L. Anchordoqui, H. Goldberg and C. Nunez, hep-ph/0408284 M. Beccaria, F. M. Renard and C. Verzegnassi, hep-ph/0412257; G. Gao, R. J. Oakes and J. M. Yang, hep-ph/0412356. 
[13] S. K. Gupta, P. Konar and B. Mukhopadhyaya, Phys. Lett. B 606, 384 (2005) hep-ph/0408296; E. J. Chun and S. C. Park, JHEP 0501, 009 (2005) hep-ph/0410242;

C. H. Chen and C. Q. Geng, hep-ph/0501001; hep-ph/0502246.

[14] C. Kokorelis, hep-th/0406258 U. Sarkar, hep-ph/0410104 I. Antoniadis and S. Dimopoulos, hep-th/0411032 P. C. Schuster, hep-ph/0412263.

[15] M. Drees, hep-ph/0501106.

[16] J. D. Wells, Phys. Rev. D 71, 015013 (2005) hep-ph/0411041.

[17] B. Kors and P. Nath, hep-th/0411201.

[18] D. R. T. Jones, Phys. Rev. D 25, 581 (1982); M. E. Machacek and M. T. Vaughn, Nucl. Phys. B 222, 83 (1983); ibid. 236, 221 (1984); J. E. Bjorkman and D. R. T. Jones, Nucl. Phys. B 259, 533 (1985).

[19] S. Eidelman et al. [Particle Data Group], Phys. Lett. B 592, 1 (2004).

[20] A. Pierce, Phys. Rev. D 70, 075006 (2004) hep-ph/0406144; A. Arvanitaki and P. W. Graham, hep-ph/0411376 A. Masiero, S. Profumo and P. Ullio, hep-ph/0412058.

[21] P. Gondolo, J. Edsjo, P. Ullio, L. Bergstrom, M. Schelke and E. A. Baltz, astro-ph/0211238.

[22] K. Cheung and W. Y. Keung, Phys. Rev. D 71, 015015 (2005) hep-ph/0408335; J. L. Hewett, B. Lillie, M. Masip and T. G. Rizzo, JHEP 0409, 070 (2004) hep-ph/0408248.

[23] A. Djouadi and Y. Mambrini, Phys. Lett. B 493, 120 (2000) hep-ph/0007174.

[24] L. Randall and R. Sundrum, Nucl. Phys. B 557, 79 (1999) hep-th/9810155; G. F. Giudice, M. A. Luty, H. Murayama and R. Rattazzi, JHEP 9812, 027 (1998) hep-ph/9810442.

[25] M. Dine and A. E. Nelson, Phys. Rev. D 48, 1277 (1993) hep-ph/9303230; M. Dine, A. E. Nelson and Y. Shirman, Phys. Rev. D 51, 1362 (1995) hep-ph/9408384; M. Dine, A. E. Nelson, Y. Nir and Y. Shirman, Phys. Rev. D 53, 2658 (1996) hep-ph/9507378; G. F. Giudice and R. Rattazzi, Phys. Rept. 322, 419 (1999) hep-ph/9801271.

[26] K. Cheung and C. W. Chiang, hep-ph/0501265.

[27] Y. Yamada, Phys. Rev. Lett. 72, 25 (1994) hep-ph/9308304. S. P. Martin and M. T. Vaughn, Phys. Lett. B 318, 331 (1993) hep-ph/9308222; J. Hisano and M. A. Shifman, Phys. Rev. D 56, 5475 (1997) hep-ph/9705417. 
[28] K. S. Babu, T. Enkhbat and B. Mukhopadhyaya, hep-ph/0501079.

[29] J. Hisano, H. Murayama and T. Goto, Phys. Rev. D 49, 1446 (1994).

[30] C. H. Chen, M. Drees and J. F. Gunion, Phys. Rev. D 55, 330 (1997) [Erratum-ibid. D 60, 039901 (1999)] hep-ph/9607421.

[31] Work in progress. 